\title{
Research on Automatic Coal Sorting Technology Based on Machine Vision
}

\section{Xu Changjie ${ }^{1, a}$, Zhang Yujie ${ }^{2, b}$, Feng Haimei ${ }^{3, c}$, Wang Junhong ${ }^{4}$, Du Chang ${ }^{5}$, Ding Jun ${ }^{6}$}

\author{
$1,2,3,4,5$ School of Mechanical-Electrical Engineering, North China Institute of Science and \\ Technology, Hebei, 065201, China \\ aemail:xuchangjie0902@163.com, bemail: 505480036 @qq.com, ${ }^{\circ} 543505993 @ q q . c o m$
}

Keywords: Automatic Sorting; Machine vision; Color extraction

\begin{abstract}
Automatic coal sorting technology based on machine vision has the function of analyzing whether there is material on the conveyor. Contour of image will be found when there is material on conveyor. The average of color will be calculated according to respective proportion of blue component and the red component in the contour. Comparing the average with the setting threshold judges the type of material. The technology has advantage of high efficiency, good stability, reliable, etc. The system also has the function of sending signals to sorting equipment and statistic number of various materials. The system reduces production costs in coal companies, and improves production efficiency, enhance the competitiveness of companies.
\end{abstract}

\section{Introduction}

Manually sorting coal has disadvantages of low production efficiency and high cost, poor working environment for workers [1-2]. Relay sorting coal has low stability, poor reliability, inconvenience and other shortcomings, which will reduce competitiveness of coal companies in the market [3]. Therefore automatic coal sorting technology based on machine vision is proposed, which can quickly and accurately implement automatic sorting coal by analyzing color characteristics of coal, gangue and metal. The technology has advantages of high efficiency, low cost and stability [4-5]. The system also has the function statistic number of various materials. At the same time, it will send signals to sorting equipment according to the type of material, so that sorting equipments send materials to the corresponding hopper.

\section{Components of Coal Sorting System Based on Machine Vision}

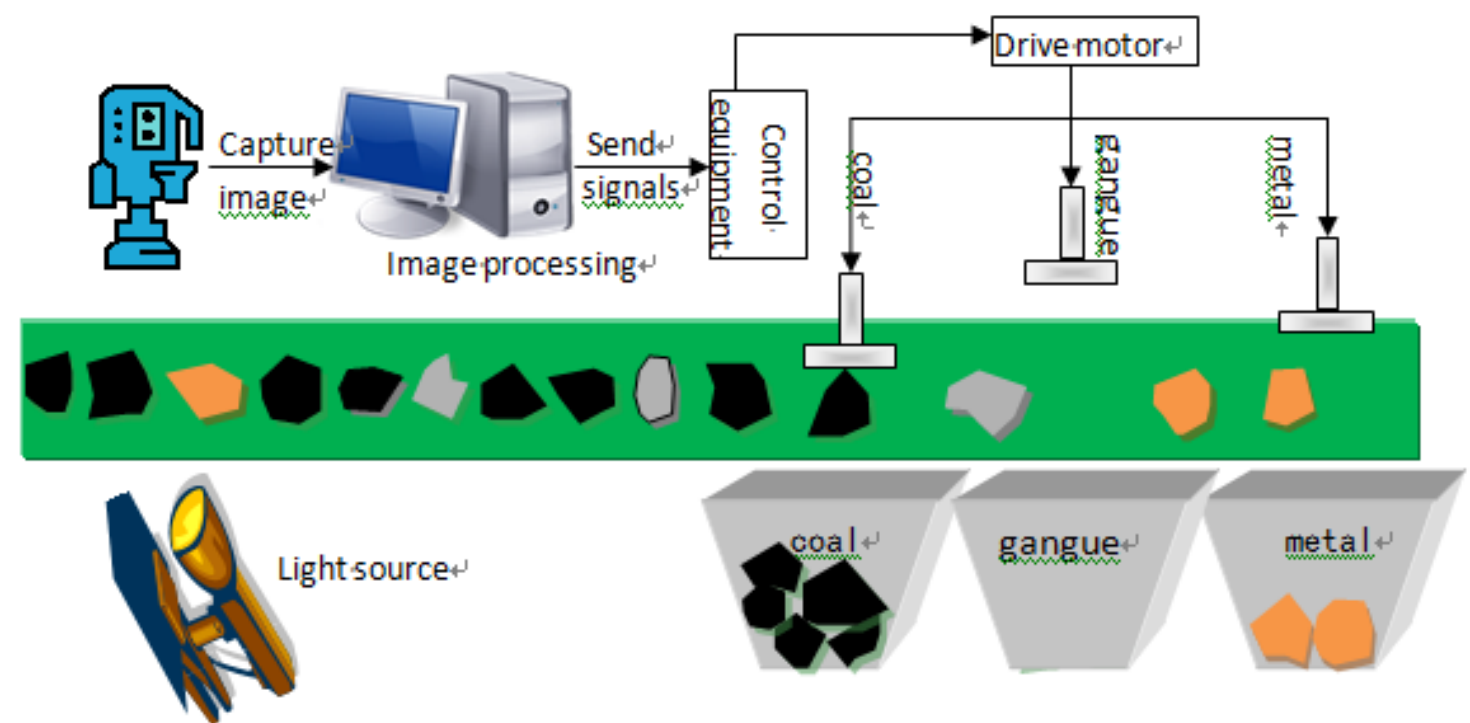

Fig. 1 Machine vision automatic coal sorting system components 
Hardware structure and relation of machine vision automatic coal sorting system in this paper is shown in Figure 1. The system consists of CMOS color industrial camera, computer, light source, conveyor and the material (including hopper), signal control equipment, drive motor and putt.

\section{Detecting Process and Analysis in Coal Sorting System Based on Machine Vision}

Detection flow chart of coal sorting system is shown in Figure.2. There are four steps:(1)setting parameters;(2)judging whether there are materials on the conveyor;(3)judging whether the material is coal;(4)judging whether the material is metal.

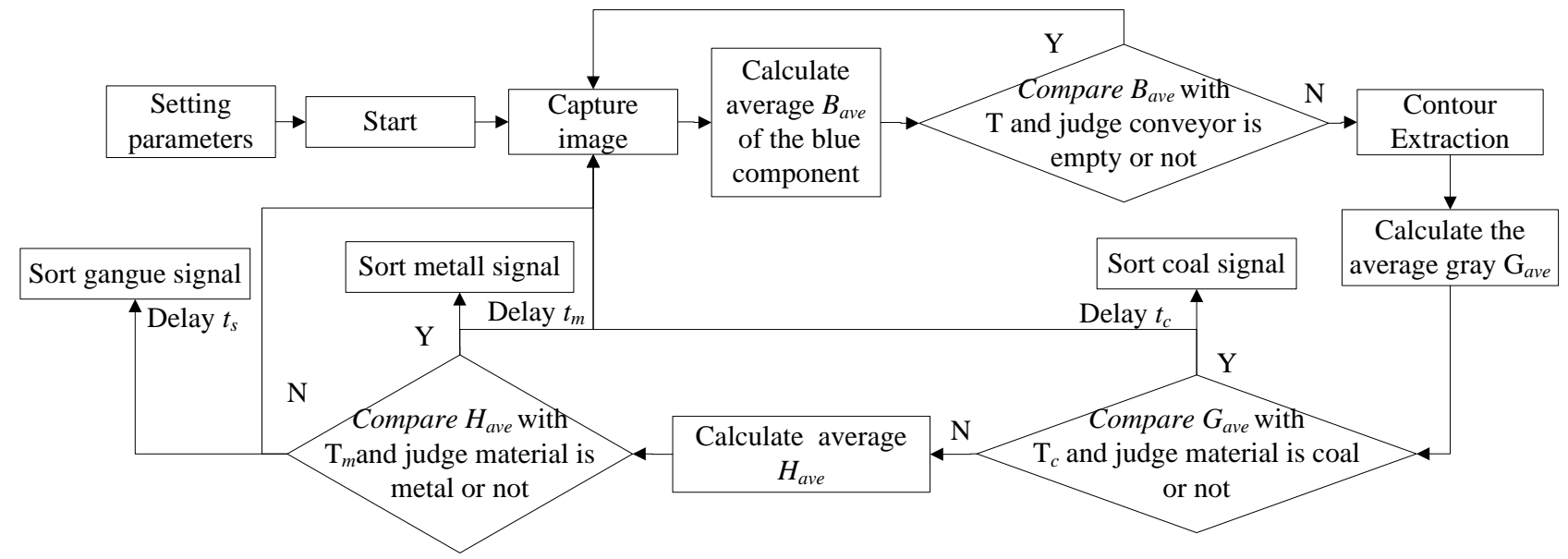

\section{Setting parameters}

Fig.2 Detection flow chart of coal sorting system

There are some parameters should be set before detecting start.

(1) Speed of the conveyor, the horizontal distance from each of the hoppers to the camera $s$ (coal $s_{c}$, gangue $s_{s}$, metal $s_{m}$ ), then according formula $t=s / v$ to calculate single delay time $t_{c} 、 t_{m}, t_{s}$.

(2) Setting yellow component (mixed with green and red) proportion $\alpha$ and red component proportion $\beta$. $\alpha=1$ when the color close to pure yellow and pure red. $\beta=1$.

(3) Setting threshold is defined range 30-210 and the default value is 100 . There are two types: a). Threshold $T_{c}$ is to judge whether material is coal or not. b). Threshold $T_{m}$ is to judge whether material is metal or not.

(4) Threshold $T$ is to judge whether conveyor is empty or not. The background of empty conveyor image is blue, so the formula of calculating $T$ is shown in Eq.(1).

$T=\frac{\sum_{i=1}^{i=m} \sum_{j=1}^{j=n} B_{i, j}}{m n}$

(1)

Here, $m$ - the number of lines in the image, $n$ - the number of column in the image, $B_{i, j}$-blue component of pixel in $i$ row and $j$ column.

\section{Judging whether conveyor is empty or not.}

Detection starts when the parameter setting is finished. The flow chart of judging whether conveyor is empty or not is shown in Figure 3.Caculating blue component average $B_{\text {ave }}$ of all pixels in image, if $0.8 T<B_{a v e}<1.2 T$, conveyor is empty and repeat this step, or there is material on the conveyor and then extract material contour on image. 


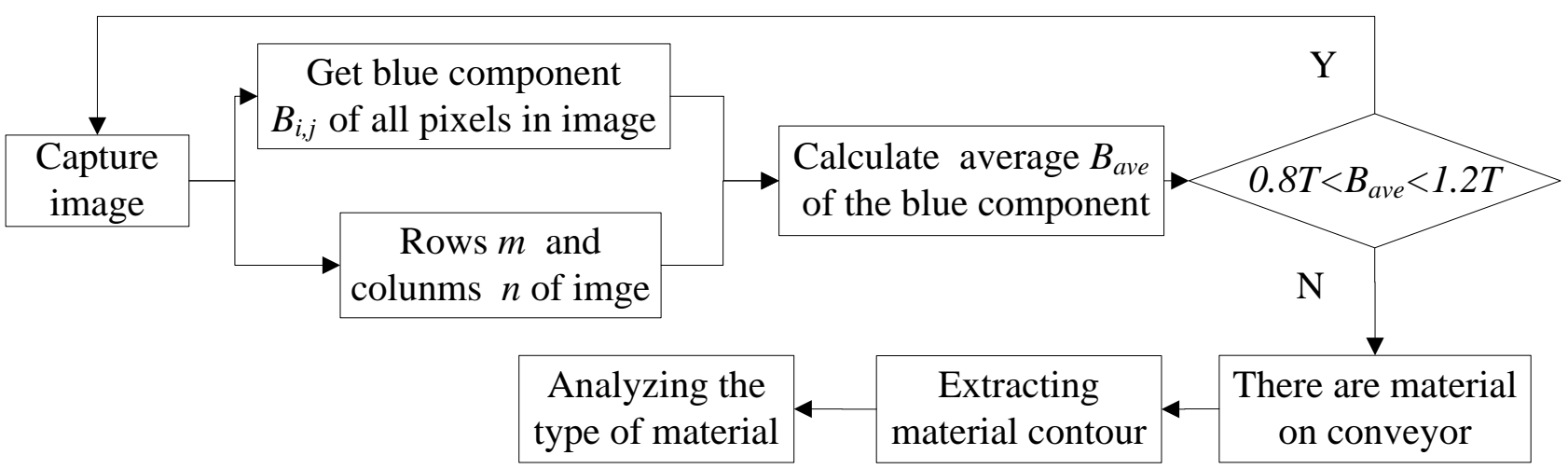

Fig.3 Flow chart of judging whether conveyor is empty or not

Extracting contour algorithm of binary image is shown in Figure 4. This paper is the need to extract the maximum contour, so contour tracking method is used to get the final contour. The basic method of contour tracing is: definite a black pixel point on the bottom left as boundary point and start to search from the top left. if the pixel point is black, it is considered as boundary point, otherwise clockwise $45^{\circ}$, until you find a black pixel as a new boundary point. Rotate counterclockwise $90^{\circ}$ based on the current search direction, using the same method to search for a boundary point, until return to the first boundary point or not find any point[3].

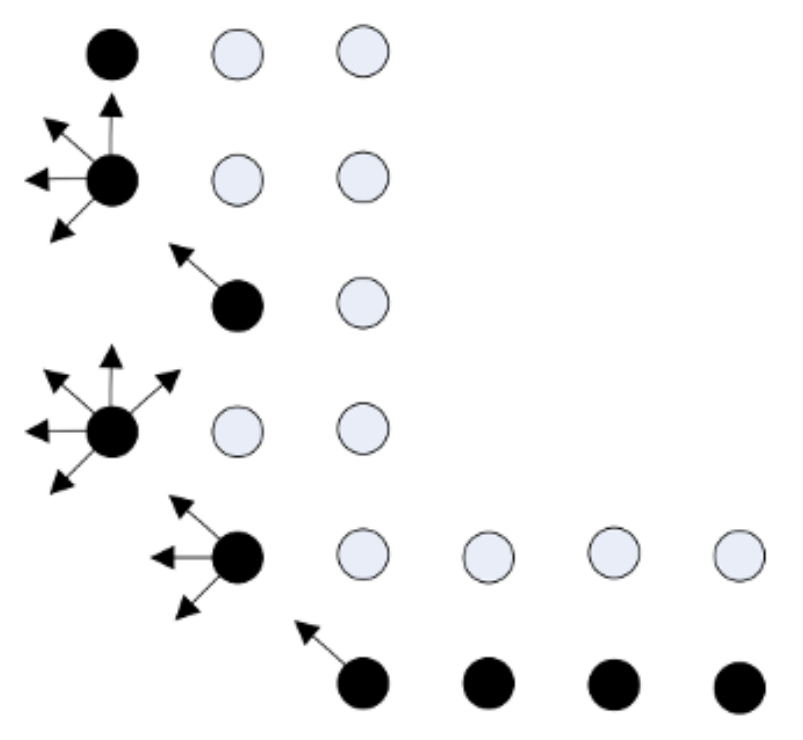

Fig.4 Schematic of contour extraction algorithm

\section{Judging the type of material}

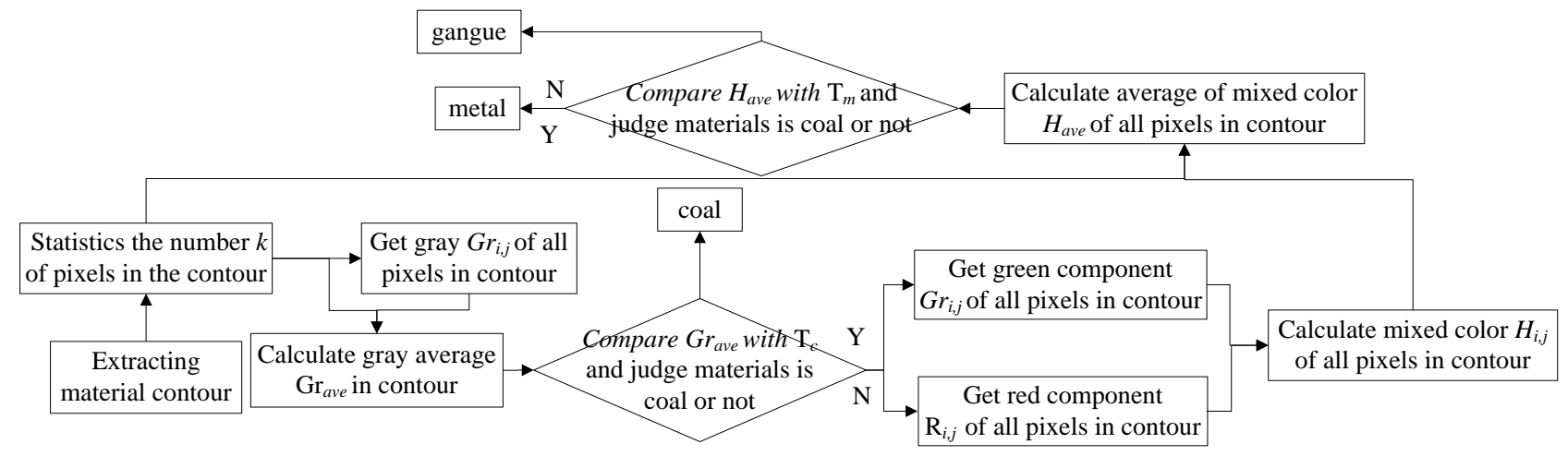

Fig.5 Flow chart of judging the type of material

Flow chart of judging the type of material is shown in Figure 5. After extracted material contour, 
statistics and records the number of pixels in contour. Calculating gray average $G r_{a v e}$ of all pixels in the contour, if $G r_{\text {ave }}<T_{c}$, material is coal and delay $t_{c}$ to send single to sort coal in coal hopper, or it is not. When material is not coal, get green component $G_{i, j}$ and red component $R_{i, j}$ to calculate mixed color $H_{i, j}$ in $i$ row and $j$ column, the formula of calculating $H_{i, j}$ is shown in Eq.(2).

$$
H_{i, j}=\alpha G_{i, j}+\beta R_{i, j}
$$

And then calculate average of mixed color $H_{a v e}$ of all pixels in contour. If $H_{a v e}>T_{m}$, material is metal and delay $t_{m}$ to send single to sort metal in metal hopper, or material is gangue and delay $t_{s}$ to send single to sort gangue in gangue hopper

\section{Conclusion}

Aimed at disadvantages of low production efficiency and high cost, poor working environment for workers of manually sorting coal and has low stability, poor reliability, inconvenience and other shortcomings of relay sorting coal in traditional companies, automatic coal sorting technology based on machine vision is proposed in this paper. This technology judge whether conveyor is empty or not and the type of material by analyzing color characteristic in image. At the same time, the system can send signal to sorting equipment and statistics number of various material. The technology has advantages of high efficiency, high stability and reliability, operational flexibility etc. The technology improve coal sorting efficiency and reduce cost.

\section{Acknowledgement}

In this paper, the research was sponsored by Youth Fund Project of North China Institute of Science and Technology (Project No. 3142014047).

\section{References}

[1]Fu qiang,Qian Jiansheng,Wang Xinhong,Song Jinling.Automatic separation of waste rock in coal mine based on image procession and recognition [J].Journal of china coal society,2000,25(5)534-537

[2]Xiang Liuzhao. Application of Automatic Coal Sorting System Based on PLC[J]. Coal Technology,2012,31(3) 54-56

[3] Liu Zhenyu ,Zhao Bin, Zou Fengshan. Applying Machine Vision Technology to Workpieces Sorting[J]. Computer Applications and Software,2012,29(11) 87-91

[4] Q.M. Zheng, W. Zhang and T.W. Yuan, The Development and Application of Intelligent Machine Vision Technology In industrial Production and Automotive Detection[J]. Manufacturing Automation. 2005, 27 411-415.

[5] S.Q. Han and L. Wang, A Survey of Thresholding Methods for Image Segmentation.[J].Systems Engineering and Electronics. 2002, 24 91-94. 